\title{
Definitions of quasiconformality
}

\author{
Juha Heinonen, Pekka Koskela
}

University of Michigan, Department of Mathematics, Ann Arbor, MI 48109, USA

Oblaturn 25-IV-1994 \& 9-VI-1994

Summary. We establish that the infinitesimal " $H$-definition" for quasiconformal mappings on Carnot groups implies global quasisymmetry, and hence the absolute continuity on almost all lines. Our method is new even in $\mathbf{R}^{n}$ where we obtain that the "limsup" condition in the $H$-definition can be replaced by a "liminf" condition. This leads to a new removability result for (quasi)conformal mappings in Euclidean spaces. An application to parametrizations of chord-arc surfaces is also given.

\section{Introduction}

A homeomorphism $f: X \rightarrow Y$ between metric spaces $X$ and $Y$ can be declared to be quasiconformal if it satisfies

$$
\limsup _{r \rightarrow 0} \frac{\sup \{|f(x)-f(y)|:|x-y|=r\}}{\inf \{|f(x)-f(y)|:|x-y|=r\}} \leqq H<\infty
$$

for all $x \in X$ and some $H$ independent of $x$. We use the distance notation $|x-y|$ and assume that our spaces have no isolated points. Generally (1.1) is a weak condition and does not lead to an interesting class of maps; for instance, a homeomorphism $f: \mathbf{R}^{1} \rightarrow \mathbf{R}^{1}$ satisfies (1.1) if it is everywhere differentiable with nonzero derivative. However, it is a fundamental fact in the theory of quasiconformal maps that if $X=Y=\mathbf{R}^{n}$ and $n \geqq 2$, then the infinitesimal condition (1.1) implies a global distortion condition, nowadays known as quasisymmetry. A homeomorphism $f: X \rightarrow Y$ is called $\eta$-quasisymmetric if there is a homeomorphism $\eta:[0, \infty) \rightarrow[0, \infty)$ such that

$$
|x-a| \leqq t|x-b| \Rightarrow|f(x)-f(a)| \leqq \eta(t)|f(x)-f(b)|
$$

Both authors supported in part by NSF. The first author also acknowledges the support of the Academy of Finland and the Sloan Foundation. 
for each $t>0$ and for each triple $x, a, b$ of points in $X$. We simply call $f$ quasisymmetric if $f$ is $\eta$-quasisymmetric for some $\eta$.

Various versions of (1.2) have been used in the literature to define quasiconformality in $\mathbf{R}^{1}$, and in spaces other than $\mathbf{R}^{n}$. Tukia and Väisälä [TV], [V2] in particular have studied quasisymmetric maps in general situations and the above definition is theirs. In his studies of rigidity of rank one symmetric spaces, Pansu [P1], [P2] defines quasiconformal maps between Carnot groups (see the definition below in Section 2) as homeomorphisms $f$ such that both $f$ and $f^{-1}$ satisfy (1.2) locally. This definition, which is a priori stronger than (1.1), was required in [P1] to prove the important "absolute continuity on lines" or ACL property of quasiconformal maps on Carnot groups. This was also the case in Mostow's celebrated work [M3], where quasiconformal maps in a non-Riemannian setting first appeared. [Mostow in fact defines quasiconformality by (1.1) but never uses this weaker definition as the maps in his situation automatically satisfy (1.2) locally; see [M3, p. 161-163] and especially formulas (21.10), (21.18) there.]

The proof of the fact that (1.1) implies (1.2) in $\mathbf{R}^{n}$ is based on the Rademacher-Stepanov theorem and appropriate capacity estimates; the crucial point is that enough analytic information (ACL, that is) can be extracted from (1.1) to perform change of variables, which then leads to a quasiinvariance of the conformal capacity. In the case $n=2$, this problem was completely settled by Gehring in [G1], and later both Gehring and Väisälä extended the result for all $n \geqq 2$. See [V1] for a complete discussion. A similar program faces formidable technical difficulties on general Carnot groups, due to their rather complicated non-Riemannian local geometry. Mostow showed recently in [M4] that (1.1) implies the ACL property for homeomorphisms on Carnot groups which are the Iwasawa components of semisimple rank one groups; these are the groups associated with the complex, quaternionic and Cayley hyperbolic spaces. Consequently, in these cases we obtain quasisymmetry (1.2) as a result of (1.1). For a thorough treatment of quasiconformal maps on the Heisenberg group, we refer to [KR].

It has been an open problem whether it is possible to conclude the ACL property of homeomorphisms satisfying (1.1) on general Carnot groups. In $[\mathrm{M} 4, \S 4]$ Mostow specifically conjectures that the answer to this question is yes, and the main purpose of this paper is to establish this conjecture. In fact, we present an elementary combinatorial argument which avoids the questions of differentiability and directly proves the equivalence of (1.1) and (1.2) for homeomorphisms of an arbitrary Carnot group $G$.

1.3. Theorem. Let $f: G \rightarrow G$ be a homeomorphism of a Carnot group $G$ onto itself. If $f$ satisfies (1.1), then $f$ satisfies (1.2) with $\eta=\eta(H, G)$.

That (1.1) implies the ACL property for homeomorphisms on Carnot groups, follows now from Theorem 1.3 and from the results in [P1].

It is generally an open problem for which spaces (1.1) and (1.2) are equivalent. Gromov and Pansu [GP, p. 93] ask for which negatively curved 
complete simply-connected manifolds $M$ a boundary homeomorphism $f: M(\infty) \rightarrow M(\infty)$ which is quasiconformal in the sense of $(1.1)$ is induced by a quasiisometry $F: M \rightarrow M$. Here $M(\infty)$ denotes the sphere at infinity equipped with a natural "Margulis conformal structure". Theorem 1.3 shows that this happens if $M(\infty)$ has a realization as a Carnot group. Heintze [H2] has classified homogeneous spaces with this property. See also 4.4 below.

Our approach to Theorem 1.3 should have some independent interest also in the classical Euclidean case, for as far we are aware, there is no previous proof of the equivalence of (1.1) and (1.2) that avoids the analytic machinery. Furthermore, in $\mathbf{R}^{n}$ the argument allows us to replace "lim sup" with "lim inf" in (1.1); this seems to be a new observation and it came as a surprise to us.

\subsection{Theorem. Let $f: \mathbf{R}^{n} \rightarrow \mathbf{R}^{n}$ be a homeomorphism, $n \geqq 2$. Suppose that}

$$
\liminf _{r \rightarrow 0} \frac{\sup \{|f(x)-f(y)|:|x-y|=r\}}{\inf \{|f(x)-f(y)|:|x-y|=r\}} \leqq H<\infty
$$

for all $x \in \mathbf{R}^{n}$ and for some $H$ independent of $x$. Then $f$ satisfies (1.2) with $\eta=\eta(n, H)$. In particular, $f$ is quasiconformal.

We do not know whether (1.5) suffices for quasisymmetry on general Carnot groups. Our argument fails in the absence of appropriate covering theorems.

Because the inverse of a quasisymmetric map is easily seen to be quasisymmetric, we obtain a new and a short proof of the fact that if $f: \mathbf{R}^{n} \rightarrow \mathbf{R}^{n}$ is quasiconformal, so is its inverse $f^{-1}$. Traditionally, it takes some effort to prove this result; see [V1].

As the issues of differentiability and change of variables are removed, we can further relax the conditions in Theorems 1.3 and 1.4 and replace the target by a more general metric space $Y$. We shall postulate two conditions on $Y$ and show that neither of them can be dropped if we are to obtain analogous results. Here, and hereafter, $B(z, r)$ denotes a closed ball in a metric space, centered at $z$ and of radius $r$. For a set $A$ in a metric space, int $A$ denotes its interior.

A metric space $Y$ is said to be $c$-linearly locally connected if there is $c \geqq 1$ such that for each $y \in Y$ and $R>0$ any two points in $Y \backslash$ int $B(y, R)$ belong to the same component of $Y \backslash$ int $B(y, R / c)$ and any two points in $B(y, R)$ belong to the same component $B(y, R c)$.

Next, assume that $\mu$ is a measure on $Y$. We say that $\mu$ is $p$-regular if there is a positive real number $p$ and a constant $C_{\mu} \geqq 1$ such that

$$
C_{\mu}^{-1} R^{p} \leqq \mu B_{R} \leqq C_{\mu} R^{p}
$$

for each ball $B_{R}$ of radius $R$ in $Y$.

Each Carnot group $G$ is both 1-linearly locally connected and carries a $Q$-regular measure, where $Q$ is the homogeneous dimension of $G$ (see 2.1). Thus Theorems 1.4 and 1.3 are corollaries of the following result: 
1.7. Theorem. Let $G$ be a Carnot group of homogeneous dimension $Q$ and let $Y$ be a c-linearly locally connected metric space that carries a $Q$-regular measure $\mu$. If a homeomorphism $f: G \rightarrow Y$ satisfies (1.1), then $f$ satisfies (1.2) with $\eta=\eta\left(G, H, c, C_{\mu}\right)$. If $G=\mathbf{R}^{n}$, it suffices to assume that $f$ satisfies (1.5).

Theorem 1.7 is new even if we used (1.1) instead of (1.5) in $\mathbf{R}^{n}$; the known analytic proofs do not allow for a general metric space as a target. Both conditions placed on $Y$ in Theorem 1.7 are necessary in the following sense. First Väisälä [V2, Section 5] has exhibited an embedding $f: \mathbf{R}^{n} \rightarrow \mathbf{R}^{n+1}$, $n \geqq 2$, such that (1.1) is satisfied but $f$ is not quasisymmetric in any neighborhood of the origin; moreover, the image $Y=f\left(\mathbf{R}^{n}\right)$ admits an $n$-regular measure, which is the restriction to $Y$ of the $n$-dimensional Hausdorff measure in $\mathbf{R}^{n+1}$. Second, in 4.7 below we show that the linear local connectivity of $Y$ alone is not sufficient for the equivalence of (1.1) and (1.2), at least if a quantitative statement akin to Theorem 1.7 is required.

The paper is organized as follows. Section 2 is preparatory and we introduce the concept of discrete modulus, which plays a crucial role in the proof of the main theorem 1.7 in Section 3. In Section 4 we give three applications: First we establish a new removability theorem for quasiconformal maps in $\mathbf{R}^{n}$ by using Theorem 1.4 ; then we show how Theorem 1.7 can be used in deciding whether a given complete, simply connected manifold of negative sectional curvature is quasi-isometric to real hyperbolic space; finally, we point out how Theorem 1.7 can be used to give a simple proof for a result of Semmes on the parametrization of chord arc surfaces. Section 5 is devoted to the proof of Proposition 2.4.

1.8. Remarks. (a) The condition we really will be using in the proof of Theorem 1.7 is

$$
\limsup _{r \rightarrow 0} \frac{(\operatorname{diam} f(B(x, r)))^{Q}}{\mu f(B(x, r))} \leqq H<\infty,
$$

which is a priori a weaker requirement than (1.1). Similarly, if $G=\mathbf{R}^{n}$, we can replace "limsup" by "liminf" in (1.9).

(b) Theorem 1.7 has a local version as well: Suppose that $f: U \rightarrow U^{\prime}$ is a homeomorphism between open subsets $U$ and $U^{\prime}$ of $G$. If $f$ satisfies (1.1), then $f$ satisfies (1.2) in a neighborhood of each point in $U$. The proof of this requires only trivial modifications to the proof presented in Section 3. For notational simplicity we consider maps that are defined in all of $G$.

\section{Preliminaries}

\subsection{Carnot groups}

A Carnot group is a connected and simply connected nilpotent Lie grol:p $G$ with graded Lie algebra $g=V_{1} \oplus \cdots \oplus V_{r}$ such that $\left[V_{1}, V_{i}\right]=V_{1+i}$. We 
also assume that $\operatorname{dim} G \geqq 2$. Assuming that the generators $X_{1}, \ldots, X_{m}$ of $V_{1}$ are fixed, $G$ admits a natural left-invariant Carnot-Carathéodory metric $d_{c}(x, y)$ which is defined as the infimum of the lengths of all horizontal paths joining $x$ and $y$; the length is measured in a Riemannian metric in which $\left\{X_{i}: i=1, \ldots, m\right\}$ is orthonormal, and a path is said to be horizontal if its tangents lie in $V_{1}$. Then the Hausdorff dimension of $\left(G, d_{c}\right)$ is equal to its homogeneous dimension $Q=\sum_{i=1}^{r} i \operatorname{dim} V_{i}$ (see [M2]). Moreover, the bi-invariant Haar measure $\mu$ in $G$ can be normalized so that each ball $B_{r}$ of radius $r$ in the metric $d_{\mathrm{c}}$ has measure $r^{Q}$. We refer to [P1] and [FS] for more information about Carnot groups.

\subsection{Capacity and modulus}

Let $E$ and $F$ be two disjoint closed subsets of a Carnot group $G$. We suppose further that $E$ is compact and that the open set $U=G \backslash(E \cup F)$ is bounded. The $Q$-capacity between $E$ and $F$ in $G$ is the number

$$
\operatorname{Cap}_{Q}(E, F)=\inf \int_{G}\left|\nabla_{0} u\right|^{Q} d \mu,
$$

where $\nabla_{0} u$ denotes the horizontal gradient of $u$ and the infimum is taken over all smooth functions $u$ on $G$ with $u \mid E \geqq 1$ and $u \mid F=0$. Standard approximation procedures allow us to enlarge the pool of admissible functions: the same value $\mathrm{Cap}_{Q}(E, F)$ results if $u$ is merely assumed to be continuous with distributional horizontal derivatives in $L^{Q}$. The $Q$-capacity is conformally invariant, hence an important tool in quasiconformal analysis. See [P1], [R], and [H1].

Next, the $Q$-modulus $\operatorname{Mod}_{Q}(E, F)$ between $E$ and $F$ is defined by

$$
\operatorname{Mod}_{Q}(E, F)=\inf \int_{U} \rho^{Q} d \mu,
$$

where the infimum is over all Borel functions $\rho$ in $U$ such that

$$
\int_{\gamma} \rho d s \geqq 1
$$

for all horizontal paths $\gamma$ joining $E$ and $F$ in $U$.

It is well known that $\operatorname{Cap}_{n}(E, F)=\operatorname{Mod}_{n}(E, F)$ in $\mathbf{R}^{n}$; this was first proved by Gehring [G2]. We require an analogous result on general Carnot groups.

\subsection{Proposition. With the above notation}

$$
\operatorname{Cap}_{Q}(E, F)=\operatorname{Mod}_{Q}(E, F) .
$$

By refining Ziemer's [Z] argument in the Euclidean case, Eichmann [E] verified Proposition 2.4 on the first Heisenberg group. To reach the general "ase, both of these proofs need to be modified slightly, and to be on the safe side we outline a proof for Proposition 2.4 in Section 6 below. 


\subsection{Discrete modulus}

Let $E$ and $F$ be closed subsets of $G$ as in 2.2. We define a "discrete modulus" between $E$ and $F$ and then show that it majorizes the standard modulus. If $B$ is a ball, we use the notation $\lambda B$ for the ball which has same center as $B$ but radius dilated by $\lambda$.

Let $\mathscr{B}$ be a countable collection of closed balls contained in $U=G \backslash(E \cup F)$, let $v: \mathscr{B} \rightarrow[0, \infty)$ be a function, and let $m$ be a positive integer. Then we call the pair $(v, \mathscr{B}) m$-admissible if the following three conditions hold:

$$
\bigcup_{B \in X} B=U
$$

there is a partition of $\mathscr{B}$ into disjoint subcollections $\mathscr{B}_{1}, \ldots, \mathscr{B}_{m}$ such that

$$
\frac{1}{5} B \cap \frac{1}{5} B^{\prime}=\emptyset
$$

whenever $B, B^{\prime} \in \mathscr{B}_{i}, B \neq B^{\prime}$, and $i=1, \ldots, m$;

$$
\sum_{B \in \sigma} v(B) \geqq 1
$$

for all chains $\sigma$ in $\mathscr{B}$ joining $E$ and $F$. Here by definition a subset $\sigma$ of $\mathscr{B}$ is a chain joining $E$ and $F$ if $\bigcup_{B \in \sigma} B$ contains a connected set whose closure meets both $E$ and $F$.

The need for the middle condition (2.7) will become apparent below; the factor $\frac{1}{5}$ comes from classical covering theorems. Note that by the Besicovitch covering theorem (see e.g. [M1, 2.7]), one often has a situation where a collection of balls in $\mathbf{R}^{n}$ can be divided into $C(n)$-subcollections satisfying (2.7). However, in this paper we shall only need the cases $m=1$ or $m=2$, of which the latter occurs only if $G \neq \mathbf{R}^{n}$.

Next, for $\delta>0$ set

$$
\mathrm{d}-\operatorname{Mod}_{Q, m}^{\delta}(E, F)=\inf \sum_{B \in \mathscr{B}} v(B)^{Q},
$$

where $Q$ is the homogeneous dimension of $G$ and the infimum is taken over all $m$-admissible pairs $(v, \mathscr{B})$ such that $\operatorname{diam} B<\delta$ for each $B \in \mathscr{B}$. Then the discrete $(Q, m)$-modulus between $E$ and $F$ is the number

$$
\mathrm{d}-\operatorname{Mod}_{Q, m}(E, F)=\lim _{\delta \rightarrow 0} \mathrm{~d}-\operatorname{Mod}_{Q, m}^{\delta}(E, F) .
$$

The next proposition shows that the discrete modulus majorizes the usual modulus defined in 2.2, up to a constant.

2.9. Proposition. There is a constant $C=C(G, m) \geqq 1$ such that

$$
\operatorname{Mod}_{Q}(E, F) \leqq C \mathrm{~d}-\operatorname{Mod}_{Q, m}(E, F) .
$$


Proof. Let $(v, \mathscr{B})$ be an $m$-admissible pair for $E$ and $F$. It suffices to exhibit an admissible density $\rho$ such that

$$
\int_{\mathbf{G}} \rho^{Q} d \mu \leqq C \sum_{B \in \mathscr{B}} v(B)^{Q} .
$$

We may assume that $\operatorname{diam} B<\delta<\frac{1}{2} \operatorname{dist}(E, F)$ for each $B \in \mathscr{B}$. Set

$$
\rho(x)=\sum_{B_{1} \in B^{\prime}} a_{i} \chi_{2 B_{i}}(x),
$$

where $a_{i}=v\left(B_{i}\right) / \operatorname{diam} B_{i}$ and $\chi_{A}$ denotes the characteristic function of a set $A$. Now let $\gamma$ be a path joining $E$ and $F$ in $U$. We find

$$
\begin{aligned}
\int_{\gamma} \rho(x) d s & =\int_{\gamma} \sum_{i} a_{i} \chi_{2 B_{i}}(x) d s \geqq \int_{\gamma} \sum_{i, \gamma \cap B_{i} \neq \emptyset} a_{i} \chi_{2 B_{i}}(x) d s \\
& =\sum_{i, \gamma \cap B_{i} \neq \emptyset} a_{i} \int_{\gamma \cap 2 B_{i}} d s=\sum_{i, \gamma \cap B_{i} \neq \emptyset} a_{i} \operatorname{length}\left(\gamma \cap 2 B_{i}\right) \\
& \geqq \frac{1}{2} \sum_{i, \gamma \cap B_{i} \neq \emptyset} v\left(B_{i}\right) \geqq \frac{1}{2} .
\end{aligned}
$$

Thus $2 \rho$ satisfies (2.3). It remains to show that $(2.10)$ holds.

To this end, let $\mathscr{B}_{1}, \ldots, \mathscr{B}_{m}$ be a partition of $\mathscr{B}$ into disjoint subcollections such that (2.7) holds. We use a standard maximal function argument (see e.g. [B, Lemma 4.2] or the remark after the proof) and infer that

$$
\int_{G}\left(\sum_{B_{i} \in B_{B_{k}}} a_{i} \chi_{2 B_{1}}(x)\right)^{Q} d \mu \leqq C \iint_{G}\left(\sum_{B_{i} \in H_{k}} a_{i} \chi_{1 / 5 B_{i}}(x)\right)^{Q} d \mu \leqq C \sum_{B_{i} \in B_{k}} v\left(B_{i}\right)^{Q}
$$

for each $k=1, \ldots, m$. To achieve (2.10), we use (2.11) $m$-times and conclude that

$$
\begin{aligned}
& \int_{G} \rho^{Q} d \mu=\int_{G}\left(\sum_{B_{i} \in \mathscr{B}_{1}} a_{i} \chi_{2 B_{B}}(x)+\cdots+\sum_{B_{1} \in \not_{m}} a_{i} \chi_{2 B_{1}}(x)\right)^{Q} d \mu \\
& \leqq m^{Q-1} \sum_{k=1}^{m} \int_{G}\left(\sum_{B_{i} \in x_{k}} a_{i} \chi_{2 B_{1}}(x)\right)^{Q} d \mu \\
& \leqq C \sum_{B_{i} \in ; B} v\left(B_{i}\right)^{Q} \text {. }
\end{aligned}
$$

This proves the proposition.

The first inequality in (2.11) is an exercise to the reader given the following two hints: use the boundedness of the Hardy-Littlewood maximal operator ([FS, p. 67]) and the duality of $L^{Q}$ and $L^{Q / Q-1)}$.

2.12. Corollary. Suppose that $E$ contains a connected set joining the center of a ball $B_{r}$ to its boundary $\partial B_{r}$ and that $F$ contains a connected set joining the boundary of $\partial B_{r}$ to the complement of $B_{2 r}$. Then

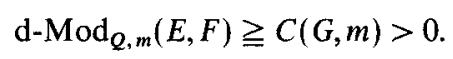


Proof. By Proposition 2.4 it suffices to have a lower bound for $\operatorname{cap}_{Q}(E, F)$. This is classical and due to Loewner [L] in the case of $\mathbf{R}^{n}$, proved by Reimann [R] in the Heisenberg group, and by the first author [H1] in general Carnot groups.

We close this section by recording, for any easy reference, the following well known covering lemma; see e.g. [FS, p. 53] or [M1, 2.1].

2.13. Covering lemma. Let $Y$ be a metric space as in Theorem 1.7 and let $A$ be a bounded subset of $Y$. Suppose that for each $x \in A$ we are given a radius $r_{x}>0$. Then there is a countable sequence of points $\left(x_{j}\right)$ in $A$ such that

$$
A \subset \bigcup_{j} B\left(x_{j}, r_{x_{j}}\right) \text { and } B\left(x_{i}, \frac{1}{5} r_{x_{1}}\right) \cap B\left(x_{j}, \frac{1}{5} r_{x_{j}}\right)=\emptyset \text { if } i \neq j \text {. }
$$

\section{Proof of Theorem 1.7}

In this section we prove Theorem 1.7. For $x \in G$ and $r>0$ we employ the following notation:

$$
L(x, r)=\sup _{|x-y|=r}|f(x)-f(y)|, \quad l(x, r)=\inf _{|x-y|=r}|f(x)-f(y)|,
$$

and

$$
H(x, r)=\frac{L(x, r)}{l(x, r)} .
$$

For notational simplicity, and obviously without loss of generality, we assume that

$$
\limsup _{r \rightarrow 0} H(x, r)<H
$$

for all $x \in G$; similarly, if $G=\mathbf{R}^{n}$, we assume that

$$
\liminf _{r \rightarrow 0} H(x, r)<H
$$

for all $x \in \mathbf{R}^{n}$.

We remark that the ensuing proof, where $Y$ is a metric space satisfying the assumptions of the theorem, is not essentially more complicated than it would be in the special case $Y=G$. The reader who is interested only in this particular situation, should choose $c=C_{\mu}=1$ throughout the proof. For those readers who are interested only in the case $Y=G=\mathbf{R}^{n}$, we shall indicate where the argument splits below.

\subsection{Preliminary steps}

To begin the proof, we first observe that both $G$ and $Y$ are $H T B$-spaces in the sense of Tukia and Väisälä [TV, 2.7]; this follows from the existence of 
a measure satisfying (1.6) together with the covering lemma 2.13 . Therefore, by an iteration argument [TV, 2.15], it suffices to verify (1.2) only for $t=1$.

Let $x_{0}, a, b \in G$ be such that $\left|x_{0}-a\right| \leqq\left|x_{0}-b\right|$, and write $L=\left|f\left(x_{0}\right)-f(a)\right|, l=\left|f\left(x_{0}\right)-f(b)\right|$. We want to show that

$$
\frac{L}{l} \leqq C<\infty,
$$

where $C=C\left(G, H, c, C_{\mu}\right)$. Because both $G$ and $Y$ are unbounded, we can pick a point $w \in G$ such that

$$
\left|x_{0}-w\right|>2\left|x_{0}-b\right| \text { and }\left|f\left(x_{0}\right)-f(w)\right|>L=\left|f\left(x_{0}\right)-f(a)\right| .
$$

Next we assume, as we may, that $L>c^{2} l$, where $c$ is the constant in the linear local connectivity condition. Now denote

$$
E=f^{-1}\left(B\left(f\left(x_{0}\right), c l\right)\right)
$$

and

$$
F=f^{-1}\left(Y \backslash \operatorname{int} B\left(f\left(x_{0}\right), L / c\right)\right),
$$

and notice that by the $c$-linear local connectedness, the closed sets $E$ and $F$ contain connected subsets joining $x_{0}$ to $b$ and $a$ to $w$, respectively. In particular, Corollary 2.12 implies

$$
\mathrm{d}-\operatorname{Mod}_{Q, m}(E, F) \geqq C(G, m)>0 .
$$

We shall show that a large ratio of $L / l$ will contradict (3.4) for $m=2$.

To do so, we need to exhibit an appropriate 2-admissible pair $(v, \mathscr{B})$ for the sets $E$ and $F$ as above.

\subsection{Description of $\mathscr{B}$}

Let $U=G \backslash(E \cup F)$ and fix $\delta>0$, to be determined later.

Suppose first that $G=\mathbf{R}^{n}$. Then for each $x \in U$ choose a radius $r_{x}$, $0<r_{x}<\delta$, such that

$$
B\left(x, 4 r_{x}\right) \subset U
$$

and that

$$
H\left(x, r_{x}\right)<H .
$$

Then from the collection $\left\{B\left(x, r_{x}\right): x \in U\right\}$ we can pick a countable subcollection $\mathscr{B}$ such that $\bigcup_{B \in \in \mathbb{B}} B=U$, that $\frac{1}{5} B \cap \frac{1}{5} B^{\prime}=\emptyset$ whenever $B, B^{\prime} \in \mathscr{B}$ and $B \neq B^{\prime}$, and that

$$
\sum_{B \in, 3 j} \chi_{B}(x) \leqq C(n)
$$

This is possible by the Besicovitch covering theorem [M1, 2.7]. Thus we have a collection $\mathscr{B}$ which satisfies (2.6) and (2.7) with $\mathscr{B}_{1}=\mathscr{B}$ and $\mathscr{B} 2=\{\emptyset$. 
It is well known that the Besicovitch covering theorem need not hold in a general Carnot group (see e.g. $[K R, 1.4]$ ) and hence our selection for $\mathscr{B}$ is more intricate in this case. The reader who is interested only in the case $G=\mathbf{R}^{n}$, may now move on to 3.17 .

Suppose $G \neq \mathbf{R}^{n}$. We divide $U$ into two subsets $\mathscr{H}$ and $\mathscr{P}$, where $\mathscr{H}$ consists of those points $x \in U$ for which

$$
\limsup _{r \rightarrow 0} \frac{\mu f(B(x, 2 r))}{\mu f(B(x, r))}<D \equiv 2\left(5 H^{2} c^{4}\right)^{Q} C_{\mu}^{2},
$$

and $\mathscr{P}=U \backslash \mathscr{H}$. We shall cover the sets $\mathscr{H}$ and $\mathscr{P}$ separately, which leads to two different subfamilies of balls. For points $x \in \mathscr{H}$ we choose a radius $r_{x}$, $0<r_{x}<\delta$, such that (3.6) holds, and, moreover, such that both

$$
H(x, r)<H
$$

and

$$
\frac{\mu f(B(x, 2 r))}{\mu f(B(x, r))}<D
$$

hold for $0<r \leqq r_{x}$. We use the covering lemma 2.13 and subtract from the family $\left\{B\left(x, r_{x}\right): x \in \mathscr{H}\right\}$ a countable subfamily $\mathscr{B}_{1}$ such that $\mathscr{H} \subset \bigcup_{B \in: B_{1}} B$ and that $\frac{1}{5} B \cap \frac{1}{5} B^{\prime}=\emptyset$ whenever $B, B^{\prime} \in \mathscr{B}_{1}$ with $B \neq B^{\prime}$.

Next we determine $\mathscr{B}_{2}$. For each $x \in \mathscr{P}$ choose a radius $r_{x}, 0<r_{x}<\delta$, such that (3.6) holds, that (3.10) holds for $0<r \leqq 2 r_{x}$, and, moreover, such that

$$
\frac{\mu f\left(B\left(x, 2 r_{x}\right)\right)}{\mu f\left(B\left(x, r_{x}\right)\right)} \geqq D / 2 .
$$

We record the following inclusions which follow from the $c$-local connectivity:

$$
B\left(f(y), \frac{1}{c} l(y, s)\right) \subset f(B(y, s)) \subset B(f(y), c L(y, s))
$$

for any $y \in G$ and $s>0$. By using this, the choice of $D$, plus the fact that

$$
C_{\mu}^{-1} s^{Q} \leqq \mu B(y, s) \leqq C_{\mu} s^{Q},
$$

we obtain for points $x \in \mathscr{P}$ that

$$
\begin{aligned}
L\left(x, r_{x}\right)^{Q} & \leqq H^{Q} l\left(x, r_{x}\right)^{Q} \leqq(H c)^{Q} C_{\mu} \mu f\left(B\left(x, r_{x}\right)\right) \\
& \leqq \frac{(H c)^{Q} C_{\mu}}{D / 2} \mu f\left(B\left(x, 2 r_{x}\right)\right) \leqq \frac{\left(H c^{2}\right)^{Q} C_{\mu}^{2}}{D / 2} L\left(x, 2 r_{x}\right)^{Q} \\
& \leqq \frac{\left(H^{2} c^{2}\right)^{Q} C_{\mu}^{2}}{D / 2} l\left(x, 2 r_{x}\right)^{Q} \leqq \frac{1}{\left(5 c^{2}\right)^{Q}} l\left(x, 2 r_{x}\right)^{Q} .
\end{aligned}
$$


We thus find

$$
B\left(f(x), 5 c L\left(x, r_{x}\right)\right) \subset f\left(B\left(x, 2 r_{x}\right)\right)
$$

for each $x \in \mathscr{P}$.

Now we use the covering lemma 2.13 in $Y$ and subtract from the collection

$$
\left\{B\left(f(x), 5 c L\left(x, r_{x}\right)\right): x \in \mathscr{P}\right\}
$$

a countable subcollection

$$
\mathscr{B}_{\mathscr{p}}=\left\{B\left(f\left(x_{i}\right), 5 c L\left(x_{i}, r_{x_{i}}\right)\right): i=1,2, \ldots\right\}
$$

such that $f(\mathscr{P}) \subset \bigcup_{B \in, \mathscr{B},} B$ and

$$
B\left(f\left(x_{i}\right), c L\left(x_{i}, r_{x_{i}}\right)\right) \cap B\left(f\left(x_{j}\right), c L\left(x_{j}, r_{x_{j}}\right)\right)=\emptyset, \quad i \neq j .
$$

Next, for each $x_{i} \in \mathscr{P}$ as above denote by $r_{i}$ the least radius $r>0$ such that the ball $B\left(x_{i}, r\right)$ contains the set $f^{-1}\left(B\left(f\left(x_{i}\right), 5 c L\left(x_{i}, r_{x_{i}}\right)\right)\right)$. Take notice that $r_{i} \leqq 2 r_{x_{1}}$ by (3.13). Then put

$$
\mathscr{B}_{2}=\left\{B\left(x_{i}, r_{i}\right): i=1,2 \ldots\right\} .
$$

Because

$$
f\left(B\left(x_{i}, \frac{1}{5} r_{i}\right)\right) \subset f\left(B\left(x_{i}, r_{x_{i}}\right)\right) \subset B\left(f\left(x_{i}\right), c L\left(x_{i}, r_{x_{i}}\right)\right),
$$

we see from (3.14) that

$$
B\left(x_{i}, \frac{1}{5} r_{i}\right) \cap B\left(x_{j}, \frac{1}{5} r_{j}\right)=\emptyset, \quad i \neq j .
$$

Moreover, $\mathscr{P} \subset \bigcup_{B \in: B_{2}} B$ by construction. We conclude that $\mathscr{B}=\mathscr{B}_{1} \cup \mathscr{B}_{2}$ satisfies (2.6) and (2.7).

Observe the following crucial property of the families $\mathscr{B}_{1}$ and $\mathscr{B}_{2}$ :

$$
\mu f\left(B_{i}\right) \leqq C_{1} \mu f\left(\frac{1}{5} B_{i}\right), \quad B_{i} \in \mathscr{B}_{1},
$$

and

$$
\mu f\left(B_{i}\right) \leqq C_{1} \mu f\left(V_{i}\right), \quad B_{i} \in \mathscr{B}_{2},
$$

where

$$
V_{i}=f^{-1}\left(B\left(f\left(x_{i}\right), c L\left(x_{i}, r_{x_{i}}\right)\right)\right) \subset B_{i}
$$

and $C_{1}=C_{1}\left(G, H, c, C_{\mu}\right)$. Indeed, (3.15) follows from (3.11) as soon as $C_{1} \geqq D^{3}$, and to see why (3.16) holds, we estimate

$$
\begin{aligned}
\mu f\left(B_{i}\right) & \leqq \mu B\left(f\left(x_{i}\right), c L\left(x_{i}, r_{i}\right)\right) \leqq C_{\mu} c^{Q} L\left(x_{i}, r_{i}\right)^{Q} \\
& \leqq C_{\mu}(H c)^{Q} l\left(x_{i}, r_{i}\right)^{Q} \leqq C_{\mu}(H c)^{Q}\left(5 c L\left(x_{i}, r_{x_{i}}\right)\right)^{Q} \\
& \leqq C_{\mu}^{2}(5 H c)^{Q} \mu f\left(V_{i}\right),
\end{aligned}
$$

where the penultimate inequality follows from the choice of $r_{i}$. Thus (3.16) bolds if $C_{1} \geqq C_{\mu}^{2}(5 H c)^{Q}$. 
We have circumvented the possible failure of Besicovitch's theorem by introducing the sets $V_{i}$; they play the role in $\mathscr{B}_{2}$ played by the sets $\frac{1}{5} B_{i}$ for $B_{i} \in \mathscr{B}_{1}$. Besides (3.15) and (3.16), notice the further similarity:

$$
\begin{gathered}
\frac{1}{5} B_{i} \subset B_{i}, \quad \frac{1}{5} B_{i} \cap \frac{1}{5} B_{j}=\emptyset, \quad i \neq j, \quad B_{i}, B_{j} \in \mathscr{B}_{1} ; \\
V_{i} \subset B_{i}, \quad V_{i} \cap V_{j}=\emptyset, \quad i \neq j, \quad B_{i}, B_{j} \in \mathscr{B}_{2} .
\end{gathered}
$$

\subsection{Definition for $v$ and admissibility}

The next step is to define $v: \mathscr{B} \rightarrow(0, \infty)$ by

$$
v(B)=\left(\log \frac{L^{\prime}}{l^{\prime}}\right)^{-1} \frac{\operatorname{diam} f(B)}{\operatorname{dist}\left(f(B), f\left(x_{0}\right)\right)},
$$

where $L^{\prime}=L / c>l^{\prime}=c l$.

We claim that $\left(C_{2} v, \mathscr{B}\right)$ is a 2-admissible pair for $E$ and $F$, where $C_{2}=C_{2}\left(G, H, c, C_{\mu}\right)$ is some positive constant. For this, it suffices to show (2.8), as we have already verified (2.6) and (2.7).

To this end, let $\sigma$ be a chain in $\mathscr{B}$ joining $E$ and $F$. Then

$$
\begin{aligned}
\sum_{\sigma} v(B) & =\left(\log \frac{L^{\prime}}{l^{\prime}}\right)^{-1} \sum_{\sigma} \frac{\operatorname{diam} f(B)}{\operatorname{dist}\left(f(B), f\left(x_{0}\right)\right)} \\
& =\left(\log \frac{L^{\prime}}{l^{\prime}}\right)^{-1} \sum_{j \geqq 0} \sum_{\sigma_{j}} \frac{\operatorname{diam} f(B)}{\operatorname{dist}\left(f(B), f\left(x_{0}\right)\right)},
\end{aligned}
$$

where $\sigma_{j}$ consists of those $B \in \sigma$ for which $f(B)$ either lies in the annular region $B\left(f\left(x_{0}\right), 2^{-j} L^{\prime}\right) \backslash B\left(f\left(x_{0}\right), 2^{-j-1} L^{\prime}\right)$ or touches $\partial B\left(f\left(x_{0}\right), 2^{-j} L^{\prime}\right)$.

At this point we make a restriction on $\delta$; we choose $\delta>0$ so small that for $B \subset U, \operatorname{diam} B<2 \delta$ implies $\operatorname{diam} f(B)<2^{-j_{0}-1} L^{\prime}$, where $j_{0}$ is the smallest integer such that $2^{-j_{o}} L^{\prime}<l^{\prime}$. In particular this means that $\left\{\sigma_{j}\right\}$ forms a partition of $\sigma$.

For the rest of the proof, we use the notation $A \geq A^{\prime}$ to indicate that $C A \geqq A^{\prime}$ for some constant $C=C\left(G, H, c, C_{\mu}\right)$.

All said, we obtain

$$
\begin{aligned}
\sum_{\sigma} v(B) & \geq\left(\log \frac{L^{\prime}}{l^{\prime}}\right)^{-1} \sum_{j \geqq 0} \sum_{\sigma_{j}}\left(2^{-j} L^{\prime}\right)^{-1} \operatorname{diam} f(B) \\
& \succeq\left(\log \frac{L^{\prime}}{l^{\prime}}\right)^{-1} \sum_{j \geqq 0}\left(2^{-j} L^{\prime}\right)^{-1} \sum_{\sigma_{j}} \operatorname{diam} f(B) \\
& \geq\left(\log \frac{L^{\prime}}{l^{\prime}}\right)^{-1} \sum_{j=0}^{j_{0}}\left(2^{-j} L^{\prime}\right)^{-1} 2^{-j} L^{\prime} \geq 1,
\end{aligned}
$$

which shows that $\left(C_{2} v, \mathscr{B}\right)$ is 2-admissible for some appropriate constant $C_{2}$ 


\subsection{Final step}

To finish the proof, we estimate the $\operatorname{sum} \sum_{B A} v(B)^{Q}$. Our choices (3.7) and (3.10) for the radii of the balls $B$ in $\mathscr{B}$ guarantee that

$$
\begin{aligned}
\sum_{B} v(B)^{Q} & =\left(\log \frac{L^{\prime}}{l^{\prime}}\right)^{-Q} \sum_{j \geqq 0} \sum_{\not^{\prime}} \frac{(\operatorname{diam} f(B))^{Q}}{\left(\operatorname{dist}\left(f(B), f\left(x_{0}\right)\right)\right)^{Q}} \\
& \leq\left(\log \frac{L^{\prime}}{l^{\prime}}\right)^{-Q} \sum_{j \geqq 0}\left(2^{-j} L^{\prime}\right)^{-Q} \sum_{B^{\prime}} \mu f(B),
\end{aligned}
$$

where $\mathscr{B}^{j}$ consists of those $B \in \mathscr{B}$ for which $f(B)$ either lies in the annular region $B\left(f\left(x_{0}\right), 2^{-j} L^{\prime}\right) \backslash B\left(f\left(x_{0}\right), 2^{-j-1} L^{\prime}\right)$ or touches $\partial B\left(f\left(x_{0}\right), 2^{-j} L^{\prime}\right)$. To deal with the second sum above, we use (3.15) and (3.16) together with disjointness of the families $\frac{1}{5} \mathscr{B}_{1}$ and $\left\{V_{i}\right\}$ :

$$
\begin{aligned}
\sum_{\boldsymbol{B}^{\prime}} \mu f(B) & \leqq \sum_{B^{\prime} \cap B_{1}} \mu f(B)+\sum_{B^{\prime} \cap B_{2}} \mu f(B) \\
& \leq \sum_{B^{\prime} \cap B_{1}} \mu f\left(\frac{1}{5} B\right)+\sum_{B^{\prime} \cap B_{2}} \mu f\left(V_{i}\right) \\
& \left.\leq \mu B\left(f\left(x_{0}\right), 2^{-j} L^{\prime}\right)\right) \preceq\left(2^{-j} L^{\prime}\right)^{Q} .
\end{aligned}
$$

Note that the finite overlapping condition (3.8) guarantees that (3.19) holds in $\mathbf{R}^{n}$ as well with $\mathscr{B}_{2}=\{\emptyset\}$.

By combining the last two estimates we arrive at

$$
\sum_{B} v(B)^{Q} \leq\left(\log \frac{L^{\prime}}{l^{\prime}}\right)^{-Q} \sum_{j=0}^{j_{0}}\left(2^{-j} L^{\prime}\right)^{-Q}\left(2^{-j} L^{\prime}\right)^{Q} \leq\left(\log \frac{L^{\prime}}{l^{\prime}}\right)^{1-Q} .
$$

In conclusion, by letting $\delta \rightarrow 0$, we find

$$
\mathrm{d}-\operatorname{Mod}_{Q, 2}(E, F) \leq\left(\log \frac{L^{\prime}}{l^{\prime}}\right)^{1-Q},
$$

which contradicts (3.4), provided the ratio $L^{\prime} / l^{\prime}$ is large enough. The theorem follows.

\section{Three applications}

\subsection{Removability theorem}

We apply Theorem 1.3 and prove the following result, which may be new even for conformal maps in the plane.

4.2. Theorem. Suppose that $f$ is a quasiconformal map of the complement of a closed set $E$ in $\mathbf{R}^{n}$ into $\mathbf{R}^{n}, n \geqq 2$, and suppose that each point $x \in E$ has the following property: there is a sequence of radii $r_{j}, r_{j} \rightarrow 0$ as $j \rightarrow \infty$, such that the annular region $B\left(x, a r_{j}\right) \backslash B\left(x, r_{j} / a\right)$ does not meet $E$ for some $a>1$ independent 
of $x$. Then $f$ has a quasiconformal extension to $\mathbf{R}^{n} \cup\{\infty\}$. Moreover, the dilatation of the extension agrees with the dilatation of $f$.

Proof. First we observe that $f$ has a homeomorphic extension to $\mathbf{R}^{n} \cup\{\infty\}$; see [MN, Theorem 1]. We call the extension $f$ as well. By Theorem 1.3 it then suffices to show that (1.5) holds for each $x \in \mathbf{R}^{n} \backslash f^{-1}(\infty)$ (note that one point is a removable singularity for quasiconformal maps). This is clear for $x$ not in $E$, so pick a point $x \in E$. Let $r_{j}$ be a radius such that $B\left(x, a r_{j}\right) \backslash B\left(x, r_{j} / a\right) \subset \mathbf{R}^{n} \backslash E$. Then

$$
\sup _{|y-x|=r,}|f(y)-f(x)| \leqq C \inf _{|y-x|=r,}|f(y)-f(x)|,
$$

where $C \geqq 1$ depends only on $a, n$ and the dilatation of $f$ in $\mathbf{R}^{n} \backslash E$. Inequality (4.3) can be proved via a standard modulus argument, or, alternatively, by observing that the quasihyperbolic diameter of the sphere $\partial B\left(x, r_{j}\right)$ in the domain int $B\left(x, a r_{j}\right) \backslash B\left(x, r_{j} / a\right)$ is bounded by a constant depending only on $a$ and then using the uniform continuity of quasiconformal maps in the quasihyperbolic metric. We leave this to the reader. In any case, $C$ is independent of $r_{j}$ and $x$, and by letting $r_{j} \rightarrow 0$ we infer that (1.5) holds everywhere in $\mathbf{R}^{n}$. Thus $f$ is quasiconformal in all of $\mathbf{R}^{n}$. On the other hand, because $E$ clearly has zero $n$-measure (no point of $E$ can be a point of density), the dilatation of the extension does not exceed the dilatation of the original map $f$ (see [V1, Chapter 4]). The theorem follows.

By the aid of Theorem 4.2 (cf. Remarks 1.8 (b)) one easily constructs Cantor type sets in $\mathbf{R}^{n}$ whose Hausdorff dimension is equal to $n$ and which are removable for all quasiconformal maps. Astala and the second author have previously pointed out that there exist compact sets of Hausdorff dimension $n$ in $\mathbf{R}^{n}$ which are similarly removable; see [AK, Example 2.5]. The condition given in Theorem 4.2 is unrelated to the example in $[\mathrm{AK}]$. For recent results on removable sets for conformal maps, see the paper by He and Schramm [HS].

\subsection{Remark on manifolds with negative curvature.}

Suppose that $M$ is a complete simply connected Riemannian manifold, of dimension at least three, with sectional curvature bounded from above by a negative constant. Then $M$ is a Gromov hyperbolic space and the sphere at infinity $M(\infty)$ has a natural conformal structure determined by metric balls. We refer to [GP] for both an excellent discussion on these matters and some of the terminology, and to $[\mathrm{GH}]$ for facts about Gromov hyperbolic spaces. Now for each $p \in M$, there is a natural homeomorphism $\varphi$ from the unit sphere $S_{p}$ in $T_{p} M$ onto $M(\infty)$, and one can ask when $\varphi$ is quasiconformal or quasisymmetric, or, rather, quasimöbius because we are working on a sphere where a four-point condition is more natural than the three-point condition (1.2). To use Theorem 1.7, we first perform a conformal "stereographic 
projection" from $M(\infty) \backslash\{a\}$ onto an unbounded space $M(\infty, a)$, where $a$ is a fixed point in $M(\infty)$ (see [GH, Chapitre 8]). Call this map $\psi_{a}$. Then if $\pi_{a}$ denotes the usual stereographic projection from $S_{p} \backslash\left\{\varphi^{-1}(a)\right\}$ onto $\mathbf{R}^{n-1}$, we obtain a map $\hat{\varphi}=\psi_{a} \circ \varphi \circ \pi_{a}^{-1}: \mathbf{R}^{n-1} \rightarrow M(\infty, a)$, and clearly $\hat{\varphi}$ satisfies (1.1) if and only if $\varphi$ does (in respective metrics).

Now if $\varphi$ is quasimöbius, it extends to a quasi-isometry between the ball model of the real hyperbolic space and $M$ (see [GP, p. 91-93], and also [P3]). Thus we obtain from Theorem 1.7 the following result.

4.5. Theorem. Suppose that $M(\infty, a)$ carries an $(n-1)$-regular measure and is linearly locally connected. Then if $\varphi$ satisfies (1.5), $M$ has to be quasi-isometric to the real hyperbolic space.

The existence of measures on the boundary of a hyperbolic space has been studied by Coornaert $[\mathrm{C}]$.

\subsection{Parametrizing surfaces}

Suppose that $E$ is a smooth simply-connected 2-dimensional surface in $\mathbf{R}^{3}$ such that $E \cup\{\infty\}$ is a smooth submanifold of $\mathbf{R}^{3} \cup\{\infty\} \approx \mathbf{S}^{3}$. Then $E$ is a chord arc surface if there is a constant $C_{E}>0$ such that

$$
C_{E}^{-1} R^{2} \leqq \mathscr{H}^{2}(E \cap B(x, R)) \leqq C_{E} R^{2}
$$

for all $x \in E$ and $R>0$, where $\mathscr{H}^{2}$ denotes the Hausdorff 2-measure, and if the two complementary components of $E$ in $\mathbf{R}^{3}$ are so-called uniform domains. See [S], [DS].

Now $E$ inherits a Riemannian metric from $\mathbf{R}^{3}$ and by Gauss's theorem there is a conformal map $f: \mathbf{R}^{2} \rightarrow E$. One can show that the internal metric on $E$ and the metric $E$ inherits from $\mathbf{R}^{3}$ are bi-Lipschitz equivalent; moreover, due to the uniformity of the complementary components of $E$, it is not hard to see that $E$ satisfies a linear local connectivity condition as described in the introduction. Because the conformal map $f$ obviously satisfies (1.1), we can conclude from Theorem 1.7 that $f$ is quasisymmetric in the Euclidean metrics of $\mathbf{R}^{2}$ and $\mathbf{R}^{3}$; moreover, the quasisymmetry function $\eta$ depends only on the chord arc constants associated with $E$, and not on the smoothness.

That the uniformizing map $f$ is globally quasisymmetric was proved by Semmes in [S] for surfaces with small constants. Later David and Semmes [DS] showed that a general smooth 2-dimensional chord-arc surface as above satisfies the crucial properties needed in the proof in [S], and hence parametrizations were found in general. We find the above argument substantially simpler than that given in [S] and [DS]. It is an open problem whether the a priori smoothness assumption for 2-dimensional surfaces can be dropped. Very recently Semmes found examples of nonsmooth 3-dimensional chord-arc surfaces in $\mathbf{R}^{4}$ which do not admit quasisymmetric parametrizations in $\mathbf{R}^{3}$ as well as smooth such surfaces which do not admit 
quasisymmetric parametrizations in $\mathbf{R}^{3}$ with constants depending only on the chord-arc structure.

\subsection{Example}

We can use the uniformization theorem as above to construct examples which show that in Theorem 1.7 condition (1.6) cannot be dropped even if we retain the linear local connectedness of the target space $Y$. To see this, consider a sequence of smooth surfaces $Y_{i}$ in $\mathbf{R}^{3}$ of the form $Y_{i}=\mathbf{R}^{1} \times \Gamma_{i}$, where $\Gamma_{i}$ is the graph of a smooth function $h_{i}: \mathbf{R}^{1} \rightarrow \mathbf{R}^{1}$. We choose $h_{i}$ such that the resulting surface $Y_{i}$ is linearly locally connected with a constant that does not depend on $i$ and that

$$
\operatorname{limsuplength}_{i \rightarrow \infty}([0,1])=\infty .
$$

We also assume that $Y_{i}$ is uniformized by the plane. Then any conformal map $f_{i}: \mathbf{R}^{2} \rightarrow Y_{i}$ satisfies (1.1) with a constant independent of $i$, but the forced asymmetry of $Y_{i}$ prevents $f_{i}$ from being quasisymmetric with a function $\eta$ that is independent of $i[\mathrm{~V} 3]$.

\section{Appendix: Proof of Proposition 2.4}

We use the notation of Section 2. First, the proof of the inequality

$$
\operatorname{Mod}_{Q}(E, F) \leqq \operatorname{Cap}_{Q}(E, F)
$$

is straightforward: Pick $u \in C_{0}^{\infty}(U)$ such that $u \geqq 1$ on $E$ and pick a rectifiable path $\gamma ;[0,1] \rightarrow \bar{U}$ joining $E$ and $F$. It is easily seen that $u \circ \gamma$ is Lipschitz on $[0,1]$, whence

$$
1 \leqq|u \circ \gamma(0)-u \circ \gamma(1)| \leqq \int_{0}^{1}\left|\nabla_{0} u(\gamma)\right||\dot{\gamma}| d t=\int_{\gamma}\left|\nabla_{0} u\right| d s .
$$

Thus $\rho=\left|\nabla u_{0}\right|$ satisfies (2.3), and (5.1) follows. (Above we need the fact, proved by Pansu in [P1, Proposition 4.1], that a locally rectifiable path $c$ : $\mathbf{R} \rightarrow G$ is almost everywhere differentiable.)

The reverse inequality is trickier. Fix $\rho$ satisfying (2.3). By the VitaliCaratheodory theorem any function $f$ in $L^{p}(U)$ can be approximated by a lower semicontinuous function $g \geqq f$ in $L^{p}(U)$ with arbitrary precision. Thus we may assume that $\rho$ is lower semicontinuous. By considering the lower semicontinuous functions $\rho_{i}=\max \left\{\rho, i^{-1}\right\}, i=1,2, \ldots$, if necessary, we may further assume that $\rho$ is bounded away from zero in $U$. We set $\rho \equiv 0$ in $G \backslash U$ so that $\rho$ is defined everywhere on $G$.

The proof of $[Z$, Lemma 3.3] applies practically verbatim to yield the following fact: Let $\left(\beta_{i}\right)$ be a sequence of paths on $G$ with uniformly bounded 
length such that for two sequences $\left(x_{i}\right)$ and $\left(y_{i}\right), x_{i}, y_{i} \in \beta_{i}$, we have $x_{i} \rightarrow x$ and $y_{i} \rightarrow y$. Then

$$
\liminf _{i \rightarrow \infty} \int_{\beta,} \rho d s \geqq \int_{\beta} \rho d s
$$

for some path $\beta$ joining $x$ and $y$. The lower semicontinuity of $\rho$ is used here, as well as, again, [P1, 4.1].

Next, set $\rho_{k}=\min \{\rho, k\}$ for $k=1,2, \ldots$. Then $\rho_{k} \in L^{\infty}(G)$ is lower semicontinuous. Défine for $x \in G$

$$
u_{k}(x)=\inf _{\beta} \rho_{\beta} \rho_{k} d s
$$

where the infimum is taken over all paths $\beta$ meeting both $x$ and $F$. By (5.2), there exists a path $\beta_{x}$ where the infimum is attained. Pick $x, y \in \bar{U}$, and let $\beta$ be a path from $x$ to $y$. Then

$$
u_{k}(y) \leqq u_{k}(x)+\int_{\beta} \rho_{k} d s \leqq u_{k}(x)+k \text { length } \beta
$$

This shows that $u_{k}$ is (locally) Lipschitz with constant $k$. Consequently, Pansu's theorem $[\mathrm{P} 1$, p. $7-8]$ implies that the horizontal gradient $\nabla_{0} u_{k}$ exists almost everywhere on $G$. We are going to show that

$$
\left|\nabla_{0} u_{k}(x)\right| \leqq \rho_{k}(x)
$$

for almost every $x \in G$.

To this end, pick $x$ such that $\nabla_{0} u_{k}(x)$ exists and recall that

$$
\left|\nabla_{0} u_{k}(x)\right|=\sup _{X \in V_{2},|X|=1}|X u(x)|
$$

where

$$
X u(x)=\lim _{t \rightarrow 0} \frac{u(x \exp (t X))-u(x)}{t}
$$

Now fix $X \in V_{1}$ such that $|X|=1$ and denote by $I$ the family of orbits of $X$. Then $i_{X}(\mathrm{Vol})$, the interior product of $X$ and the fixed bi-invariant volume form on $G$, yields a natural measure $d \gamma$ on $\Gamma$, (cf. [P1, p. 21]). Fix a surface $S$ transversal to $\Gamma$. We can parametrize the paths in $\Gamma$ by $\gamma_{p}(t)=p \exp (t X)$, $p \in S, t \in \mathbf{R}$. By a standard argument (see [V1, Section 28]) we infer that $\rho_{k}$ is Borel measurable on almost every path $\gamma \in \Gamma$. Now it follows by a straightforward argument (cf. [P1, p. 31]) that for almost every $x \in G$ the function $\rho_{k}$ is approximately continuous at $x$, when restricted to the path $t \mapsto x \exp (t X)$. (Recall that a function $h$ is approximately continuous at a point $y_{0}$ if $h(y) \rightarrow h\left(y_{0}\right)$ when $y$ approaches $y_{0}$ along a set with measure density 1 at $y_{0}$; it is well known that bounded measurable functions on a measure space are 
approximately continuous at almost every point $[\mathrm{F}, \mathrm{p}, 159])$. Thus for all such $x$ we have that

$$
u_{k}(x \exp (t X)) \leqq u_{k}(x)+\int_{\beta_{\mathrm{r}}} \rho_{k} d s,
$$

where $\beta_{x, t}(s)=x \exp (s X), 0 \leqq s \leqq t$. It follows that

$$
\lim _{t \rightarrow 0} \frac{u(x \exp (t X))-u(x)}{t} \leqq \lim _{t \rightarrow 0} \frac{1}{t} \int_{\beta_{x}} \rho_{k} d s=\rho_{k}(x)
$$

by approximate continuity. This proves (5.4).

The rest of the proof goes exactly as in [Z]. By using (5.2) and the fact that $\rho$ is bounded away from zero, we first infer that $m_{k}=\min _{x \in E} u_{k}(x) \rightarrow 1$ as $k \rightarrow \infty$ and then that

$$
\begin{aligned}
\operatorname{Cap}_{Q}(E, F) & \leqq \liminf _{k \rightarrow \infty} m_{k}^{-Q} \int_{G}\left|\nabla_{0} \min \left\{u_{k}, m_{k}\right\}\right|^{Q} d x \\
& \leqq \liminf _{k \rightarrow \infty} m_{k}^{-Q} \int_{U} \rho_{k}^{Q} d x \leqq \int_{U} \rho_{k}^{Q} d x
\end{aligned}
$$

Because $\rho$ was arbitrary, this completes the proof.

Acknowledgements. We wish to thank Fred Gehring and Jussi Väisälä for their interest in this work and for their helpful comments on the manuscript.

\section{References}

[AK] Astala K., Koskela P.: Quasiconformal mappings and global integrability of the derivative. J. Anal, Math. 57, 203-220 (1991)

[B] Bojarski B.: Remarks on Sobolev imbedding inequalities, In Proc. of the Conference on Complex Analysis, Joensuu 1987. Lecture Notes in Math. 1351, Springer Verlag 1988

[C] Coornaert M.: Mesures de Patterson-Sullivan sur le bord d'un espace hyperbolique au sens de Gromov, Pacific J. Math. 159, 241-270 (1993)

[DS] David G., Semmes S.: Quantitative rectifiability and Lipschitz mappings, Trans. Amer. Math. Soc. 337, 855889 (1993)

[E] Eichmann R.: Variationsprobleme auf der Heisenberggruppe, Lizentiatsarbeit. Universität Bern (1990)

[F] Federer H.: Geometric Measure Theory, Springer, New York, 1969.

[FS] Folland G.B., Stein E.M.: Hardy spaces on homogeneous groups, Princeton University Press, Princeton, New Jersey, 1982

[G1] Gehring F.W.: The definitions and exceptional sets for quasiconformal mappings. Ann. Acad. Sci. Fenn. Ser. A I Math. 281, 1-28 (1960)

[G2] Gehring F.W.: Extremal length definitions for the conformal capacity of rings in space, Michigan Math. J. 9, 137-150 (1962)

[GH] Ghys E., de la Harpe P.: Sur les Groupes Hyperboliques d'après Mikhae। Gromov, Birkhäuser, Progress in Mathematics, Boston-Basel-Berlin, 1990

[GP] Gromov M., Pansu P.: Rigidity of Lattices: An Introduction, Geometric Topol ogy: Recent Developments. Lecture Notes in Mathematics 1504, Springer-Verla Berlin-New York-Heidelberg, 1991 
[HS] He Z.X., Schramm O.: Rigidity of circle domains whose boundary has $\sigma$-finite linear measure, Invent. Math. 115, 297-310 (1994)

[H1] Heinonen J.: A capacity estimate on Carnot groups, Bull. Sci. Math. Fr. (to appear)

[H2] Heintze E.: On homogeneous manifolds of negative curvature, Math. Ann. 211, 23-34 (1974)

[KR] Korányi A., Reimann H.M.: Foundations for the theory of quasiconformal mappings on the Heisenberg group, Adv. in Math. (to appear)

[L] Loewner C.: On the conformal capacity in space, J. Math. Mech. 8, 411-414 (1959)

[MN] Martio O., Näkki R.: Continuation of quasiconformal mappings, (in Russian) Sib. Mat. Zh. 28, 162-170 (1987), English translation: Siberian Math. J. 28, 645-652 (1988)

[M1] Mattila P.: Geometry of sets and measures in Euclidean spaces, to appear in Cambridge Univ. Press

[M2] Mitchell J.: On Carnot-Carathéodory metrices, J. Diff. Geom. 21, 35-45 (1985)

[M3] Mostow G.D.: Strong rigidity of locally symmetric spaces, Princeton University Press, Princeton, New Jersey, 1973

[M4] Mostow G.D.: A remark on quasiconformal mappings on Carnot groups, Michigan Math. J. 41, 31-37 (1994)

[P1] Pansu P.: Métriques de Carnot-Carathéodory et quasiisométries des espaces symétriques de rang un, Ann. Math. 129, 1-60 (1989)

[P2] Pansu P.: Dimension conforme et sphère à l'infini des veriétés à courbure négative, Ann. Acad. Sci. Fenn. Ser. A I Math. 14, 177-212 (1989)

[P3] Paulin F.: Un groupe hyperbolique est déterminé par son bord, preprint (1993)

[R] Reimann H.M.: An estimate for pseudoconformal capacities on the sphere, Ann. Acad. Sci. Fenn. Ser. A I Math 14, 315-324 (1989)

[S] Semmes S.: Chord-arc surfaces with small constant. II. Good parameterizations, Adv. in Math. 88, 170-199

[TV] Tukia P., Väisälä J.: Quasisymmetric embeddings of metric spaces, Ann. Acad. Sci. Fenn. Ser. A I Math. 5, 97-114 (1980)

[V1] Väisälä J.: Lectures on n-dimensional quasiconformal mappings, Lecture Notes in Math. 229, Springer-Verlag, Berlin-Heidelberg-New York, 1971

[V2] Väisälä J.: Quasisymmetric embeddings in euclidean spaces, Trans. Amer. Math. Soc. 264, 191-204 (1981)

[V3] Väisälä J.: Quasisymmetric maps of products of curves into the plane, Rev. Roumaine Math. Pures Appl. 33, 147-156 (1988)

[Z ] Ziemer W.P.: Extremal length and p-capacity, Michigan Math. J. 16, 43-51 (1969) 